\title{
Cutaneous Leishmaniasis Situation and Predicting the Distribution of Phlebotomus papatasi and $P$. sergenti as Vectors of Leishmaniasis in Ardabil Province, Iran
}

\author{
Ali Khamesipour', Soheila Molaei ${ }^{2}$, Navid Babaei-Pouya ${ }^{3}$, Eslam Moradi-As| ${ }^{4,5, *}$ \\ ${ }^{1}$ Center for Research and Training in Skin Diseases and Leprosy, Tehran University of Medical Sciences, Tehran, Iran; ${ }^{2}$ Deputy of Research\&Technology, \\ Ardabil University of Medical Sciences, Ardabil, Iran; ${ }^{3}$ Nir County Health Centre, Ardabil University of Medical Sciences, Ardabil, Iran; ${ }^{4}$ Department of \\ Public Health, School of Public Health, Ardabil University of Medical Sciences, Ardabil, Iran; ${ }^{5}$ Arthropod Borne Diseases Research Center, Ardabil \\ University of Medical Sciences, Ardabil, Iran
}

\begin{abstract}
Cutaneous leishmaniosis $(C L)$ is the most common form of leishmaniasis.CL caused by $L$. major and $L$. tropica is endemic in 17 provinces of Iran. This study was carried out to elucidate situation of CL in Ardabil province and to predict distribution of Phlebotomus papatasi and Phlebotomus sergenti (Diptera: Psychodidae) as vectors of CL in the region. In this cross-sectional study, data on CL patients were collected from local health centers of Ardabil province, Iran during 2006-2018 to establish a geodatabase using ArcGIS10.3. A total of $20 \mathrm{CL}$ cases were selected randomly and skin samples were collected and analyzed by PCR method. MaxEnt 3.3.3 model was used to determine ecologically suitable niches for the main vectors. A total, $309 \mathrm{CL}$ human cases were reported and the highest incidence rate of disease was occurred in Bilasavar (37/100,000) and Germi (35/100,000). A total of 2,794 sand flies were collected during May to October 2018. The environmentally suitable habitats for $P$. papatasi and $P$. sergenti were predicted to be present in northern and central areas of Ardabil province. The most variable that contributed ratio in the modeling were Isothermality and slope factors. Ardabil province is possibly an endemic are for CL. The presence of $P$. papatasi and $P$. sergenti justifies local transmission while the vectors of $\mathrm{CL}$ are existing in the northern and central areas of the province.
\end{abstract}

Key words: Phlebotomus papatasi, Phlebotomus sergenti, cutaneous leishmaniasis, vector, Iran

\section{INTRODUCTION}

Cutaneous Leishmaniasis (CL) is one of the neglected tropical disease caused by different Leishmania species which is endemic to more than 100 countries, annually 700,000-1,200,000 new cases of CL and visceral leishmaniasis (VL) in the world [1]. Four common clinical types of leishmaniasis are usually presented; CL, mucocutaneous leishmaniasis (MCL), diffuse cutaneous leishmaniasis (DCL) and VL. CL is the most common form of leishmaniasis, 2 types of CL were reported [2]. In Iran, 2 types of CL is endemic; zoonotic cutaneous leishmaniasis (ZCL) and anthroponotic cutaneous leishmaniasis (ACL) [3]. Annually, more than 20,000 cases of CL were reported in Iran. ACL is caused by Leishmania tropica and transmitted by

\footnotetext{
- Received 28 December 2019, revised 22 February 2020, accepted 19 March 2020. *Corresponding author (Moradiasl83@yahoo.com) (C) 2020, Korean Society for Parasitology and Tropical Medicine This is an Open Access article distributed under the terms of the Creative Commons Attribution Non-Commercial License (https://creativecommons.org/licenses/by-nc/4.0) which permits unrestricted non-commercial use, distribution, and reproduction in any medium, provided the original work is properly cited.
}

Phlebotomus (Para-phlebotomous)sergenti [4,5]. ACL was reported from Tehran, Mashhad, Shiraz, Kerman, Yazd, Bam and etc. $[6,7]$. In Iran, ZCL is caused by L. major and transmitted by Phlebotomus papatasi and small rodents are the main reservoir. ZCL is endemic in 17 out of 31 provinces, Iran [3,8,9]. Over 800 species of sand flies are known but only over 30 transmit Leishmania [8]. 44 spices of sand flies are reported in Iran and 10 species is doubtful [9-11]. Ardabil province is located in northwest of Iran and is the main endemic areas of VL and annually 15-20 new cases were reported $[12,13]$. To our knowledge through search on internet, no study is conducted on CL in the northwest of Iran including Ardabil Province. Previously, Leishmania parasite has been reported from 2 spices of Sergentomyia dentata and S. sintoni as well as Leishmania was isolated from $P$. papatasi using molecular methods. The parasite species has not been identified [14,15]. This study aims to explain about CL situation in Ardabil province and to explore distribution of P. papatasi and P. sergenti (Diptera:Psychodidae) as possible vectors of leishmaniasis in the province. 


\section{MATERIALS AND METHODS}

\section{Study area}

Ardabil Province is located in the north-west of Iran (37.45$39.42^{\circ} \mathrm{N}, 47.30-48.55^{\circ} \mathrm{E}$ ). The province has an area of 17,953 $\mathrm{km}^{2}$ and a population of 1,270,000, based on 2016 census. This province consists of 10 counties, 21 districts, 26 cities, 71 rural districts and 1,477 permanent villages [16].

\section{Data collection}

CL patients' data are collected from the source document of each patient from Health Center of Ardabil province from 2006 to 2018, the information in regard to demographic, travel history, site and number of lesion was recorded and analyzed. Sand flies were collected from 30 areas during May to November 2018. The study areas were selected based on the frequency of human $\mathrm{CL}$ in the past 10 years. Five urban areas and 25 villages were selected, and their geographic coordinates were recorded using Global Positioning System. Sand flies were collected using caster oiled papers (60 papers for indoors and outdoors of each area). Then, the collected sand flies were put in acetone and then stored in the 70\% alcohol. The speci- mens were mounted by addition of a single drop of Puris' medium and identified using relevant identification keys $[17,18]$.

\section{PCR method}

A sample was prepared from the slide which was used for direct smear, the skin materials were isolated from the slide by scratching, DNA was extracted and used for PCR amplification according to the procedure previously described $[19,20]$. PCR will be carried out using the primers for leishmania-specific pair fragment of kinetoplast DNA, F: (5'TCGCAGAACGCCCCTACC, $3^{\prime}$ ) and R: (5' AGGGGTTGGTGTAAAATAGG 3'). Amplification; 35 cycle, denaturation at $94^{\circ} \mathrm{C}$ for $1 \mathrm{~min}$, annealing at $60^{\circ} \mathrm{C}$ for $45 \mathrm{sec}$, extension at $72^{\circ} \mathrm{C}$ for $1 \mathrm{~min}$ and final extension at $72^{\circ} \mathrm{C}$ for $7 \mathrm{~min}$.

\section{Modeling}

The MaxEnt version 3.3.3 was used to predict the presence of $P$. papatasi and $P$. sergenti sand flies in order to prepare the distribution map at 72 locations (30 locations from P. sergenti and 42 locations for $P$. papatasi) in Ardabil Province northwest of Iran (Table 1). Jackknife test was used to analyze the relationship between weather variables and distribution of sand

Table 1. Variables used for MaxEnt modeling of cutaneous leishmaniasis distribution in Ardabil Province, northwest of Iran

\begin{tabular}{|c|c|c|c|}
\hline \multirow{2}{*}{ Variable } & \multirow{2}{*}{ Description } & \multicolumn{2}{|c|}{ Contribution (\%) } \\
\hline & & Phlebotomus papatasi & Phlebotomus sergenti \\
\hline Bio1 & Annual mean temperature $\left({ }^{\circ} \mathrm{C}\right)$ & 0 & 0 \\
\hline Bio2 & Mean diurnal range: mean of monthly (max temp-min temp) $\left({ }^{\circ} \mathrm{C}\right)$ & 0 & 0 \\
\hline Bio3 & Isothermality: (Bio2/Bio7) × 100 & 0.2 & 24.1 \\
\hline Bio4 & Temperature seasonality (SD × 100) & 0.6 & 0.4 \\
\hline Bio6 & Minimum temperature of coldest month $\left({ }^{\circ} \mathrm{C}\right)$ & 0 & 0 \\
\hline Bio7 & Temperature annual range (Bio5 - Bio6) $\left({ }^{\circ} \mathrm{C}\right)$ & 0 & 3.2 \\
\hline Bio8 & Mean temperature of wettest quarter $\left({ }^{\circ} \mathrm{C}\right)$ & 2.8 & 19.7 \\
\hline Bio9 & Mean temperature of driest quarter $\left({ }^{\circ} \mathrm{C}\right)$ & 0 & 0 \\
\hline Bio10 & Mean temperature of warmest quarter $\left({ }^{\circ} \mathrm{C}\right)$ & 0.6 & 10.2 \\
\hline Bio11 & Mean temperature of coldest quarter $\left({ }^{\circ} \mathrm{C}\right)$ & 0.8 & 0 \\
\hline Bio13 & Precipitation of wettest month (mm) & 7.6 & 0.5 \\
\hline Bio14 & Precipitation of driest month (mm) & 6.3 & 0 \\
\hline Bio15 & Precipitation seasonality (coefficient of variation) & 0.2 & 4.6 \\
\hline Bio16 & Precipitation of wettest quarter (mm) & 0.5 & 0 \\
\hline Bio17 & Precipitation of driest quarter (mm) & 1.2 & 0 \\
\hline Bio18 & Precipitation of warmest quarter (mm) & 1.1 & 0 \\
\hline Bio19 & Precipitation of coldest quarter (mm) & 0.5 & 0.5 \\
\hline Altitude & Elevation from the sea level (m) & 0 & 0 \\
\hline Slope & Slope of the area (\%) & 56.4 & 12.1 \\
\hline Aspect & direction of slope (degree) & 6.2 & 13.8 \\
\hline NDVI & -1 to +1 & 14.9 & 0 \\
\hline
\end{tabular}


flies and the relevant variables were identified with percentages and non-relevant variables were assigned zero. The output of the MaxEnt model was included in the ArcMap 10.4.1 software and was analyzed by Jackknife test and descriptive statistical.

\section{RESULTS}

\section{Aspects of cutaneous leishmaniasis}

Totally, 309 cases of CL were reported during 2005-2018 in Ardabil Province. The highest number (31cases) of CL was reported in 2009 and the lowest number (13 cases) in 2011 year (Fig. 1), CL were reported in 9 of 10 counties in Ardabil Province and the highest number was reported in Bilasavar region for 37/100,000 and Germi 35/100,000, about 50\% of CL patients were reported from the north part, 39\% from the central parts and 11\% from south parts of Ardabil Province (Fig. 2), $71 \%$ of CL were males and $29 \%$ were females, The age range of the patients was $86 \%$ over 10 years and $14 \%$ under 10 years,

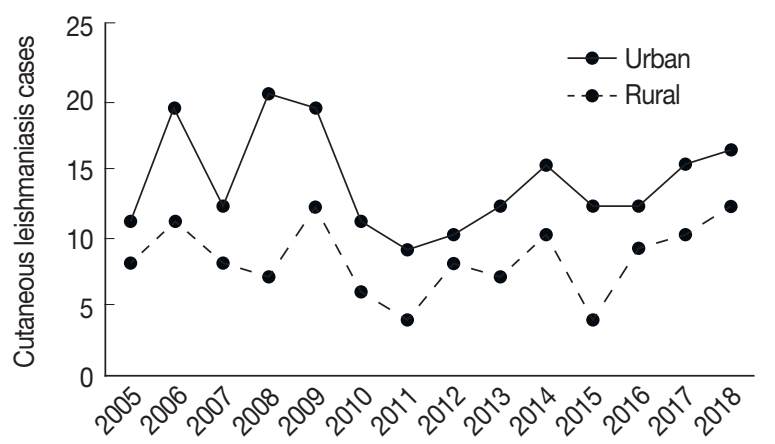

Fig. 1. Trend of $C L$ cases reported in urban and rural areas in $\mathrm{Ar}$ dabil Province, northwest of Iran (2005-2018).
A

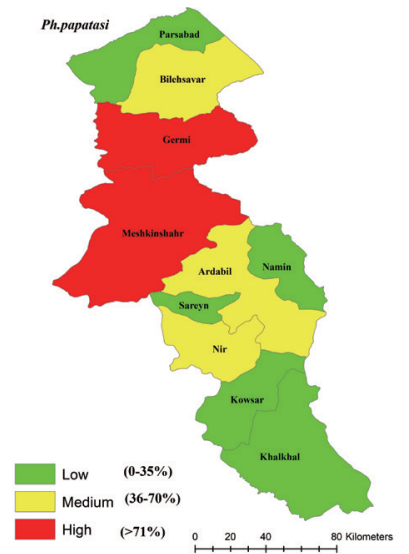

B

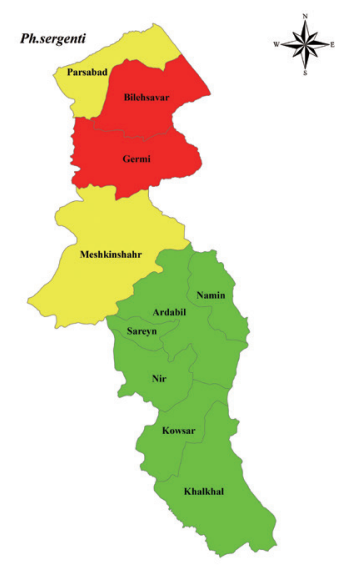

Fig. 2. Frequency of Phlebotomus papatasi (A) and Phlebotomus sergenti (B) in northwest provinces, Iran, 2018.
$53 \%$ of the patients mentioned a history of travel outside the province. Annually, 20-30 CL cases were reported from Ardabil Province which $74.5 \%$ of CL reported from 13 urban areas and $24.5 \%$ from 46 rural areas, The clinical picture of the lesions showed that $65 \%$ of the lesions were dry with no secretion and $35 \%$ of the lesions were wet with secretion, most of the lesions (35.5\%) was seen in hands and 30\% in the face (Fig. 3), 47\% of the patients present only a single lesion and 53\% more than one lesion. The PCR results showed that the causative agents were Leishmania tropica in line 7 and L. major in lanes 1, 4, and 6 (Fig. 4).

\section{Sand flies collected}

A total of 2,794 sand flies were collected from 30 locations of Ardabil province, $40 \%$ of the total sand flies were belong to

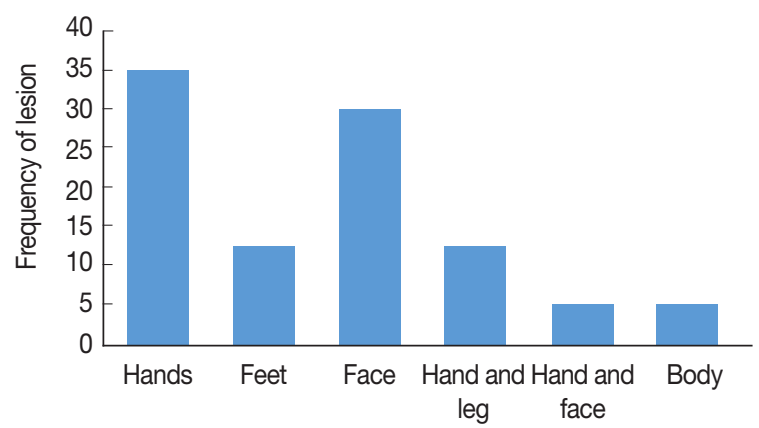

Fig. 3. The frequency and location of lesion of $C L$ in different areas, Ardabil Province, northwest Iran (2005-2018).

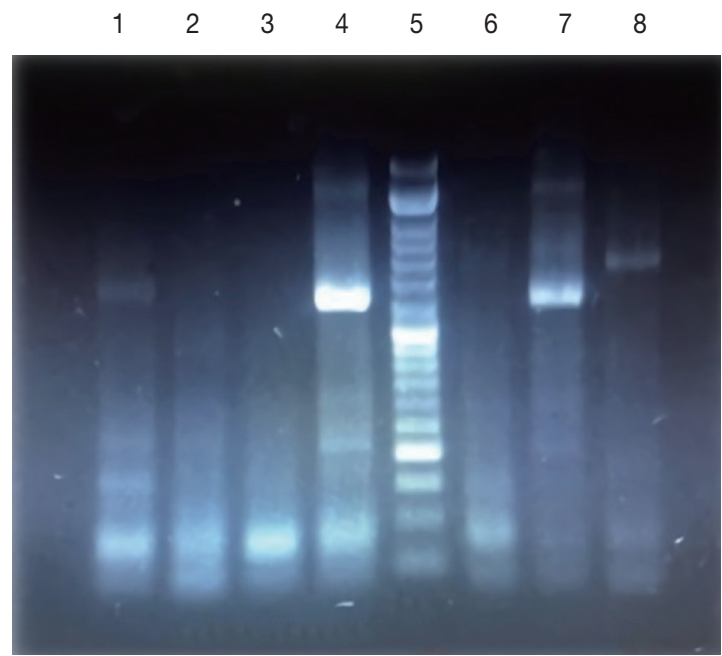

Fig. 4. 1.5\% agarose gel electrophoresis of PCR amplification for identification of Leishmania species on Giemsa-Stained slides. Lanes 1,4,7: Standard Leishmania major; Lanes 2,3,6: negative control; Lane 5: DNA Ladder (50 bp); Lane 8: Leishmania tropica. 
Table 2. Fauna and number of collected sand flies in Ardabil Province, northwest Iran, 2018

\begin{tabular}{llcrrrrr}
\hline Subgenus & \multicolumn{1}{c}{ Spices } & Total collected & Male (\%) & Female (\%) & Outdoors (\%) & Indoors (\%) & $P$-value \\
\hline Phlebotomus & Phlebotomus papatasi & 662 & 71 & 29 & 55 & 45 & $<0.05$ \\
Paraphlebotomus & Phlebotomus sergenti & 340 & 74.7 & 25.3 & 75.6 & 24.4 & $<0.05$ \\
& Phlebotomus caucasicus & 30 & 73.3 & 26.7 & 63.3 & 36.7 & $<0.05$ \\
& Phlebotomus mongolensis & 49 & 100 & 0 & 89.8 & 10.2 & $<0.05$ \\
& Phlebotomus jacusieli & 6 & 100 & 0 & 100 & 0 & $<0.05$ \\
& Phlebotomus andrejevi & 31 & 100 & 0 & 93.6 & 6.5 & $<0.05$ \\
\hline
\end{tabular}

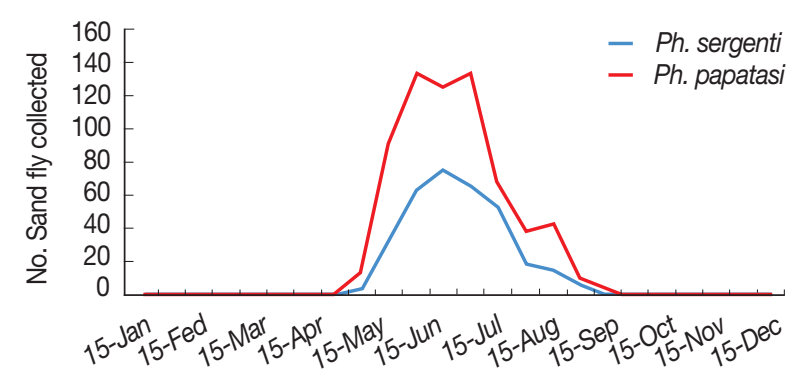

Fig. 5. Monthly activity of Phlebotomus papatasi and Phlebotomus sergenti in Ardabil province, Iran, 2018.

the 2 phlebotomus and paraphlebotomus subgenus and $60 \%$ to 6 other spices (Fig. 2). P. papatasi were collected from all of the study sites but $P$. sergenti was collected in 22 of the 30 sites.

\section{P. papatasi}

Only 1 spice of Phlebotomus subgenus was collected and $23.7 \%$ of the total collected sand flies were belong to this spices $(\mathrm{M}=71 \%, \mathrm{~F}=29 \%), 55 \%$ of $P$. papatasi were collected from outdoors and $45 \%$ from indoors (Table 2 ). The frequency of $P$. papatasi was the highest in all the study areas of Ardabil Province and the maximum number of samples were collected from in Meshkinshahr and Germy counties (Fig. 2). Monthly activity of $P$. papatasi in Ardabil province was started in 30 April and ended in 15 October (when density of sand flies reaches to zero), with one peak between in 15 June to 15 July (Fig. 5).

\section{P. sergenti}

Five spices of this Paraphlebotomus were collected in the study areas and $16.32 \%$ of total sand flies were belong to these spices $(M=74.7 \%, F=25.3 \%), 75.5 \%$ of the species were collected from outdoors and $24.5 \%$ of indoors. The highest and lowest spices in paraphlebotomus subgenus was $P$. sergenti (74.6\%) and P. jacusieli (1.3\%), respectively (Table 2). The frequency of $P$. sergenti was the highest in north and central parts and the highest number of the spices were collected from Germy and Bilasavar counties (Fig. 2). Monthly activity of $P$. sergenti in Ardabil Province was started in 15 May and ended in 30 September (when density of sand flies reaches to zero) with one peak in June (Fig. 5).

\section{Ecological niche modeling P. sergenti}

The environmental suitability for $P$. sergenti was predicted to be present in most parts of northern Ardabil Province (Fig. $6 \mathrm{~A})$, although there are some areas of high environmental suitability in northeastern, northwestern regions as well. Warmer colors show areas with higher environmental suitability for $P$. sergenti.The areas under receiver operating characteristic (ROC) curve (AUC) for training and testing data was calculated as 0.904 and 0.933 , respectively. The most variable seems to contribute the ratio in the modeling of $P$. sergenti was Bio3 (Isothermality) factor and Jackknife test results showed that the environmental variable with the highest gain in the predicting power of the model if used in isolation was Slope variable (Fig. 7A).

\section{P. papatasi}

The results of this study showed that the environmental suitability habitat for $P$. papatasi was in northern and central parts of Ardabil province (Fig. 6B). There are some areas of high environmental suitability in northern regions in borderline of Iran and Azerbaijan Republic country. The area under receiver operating characteristic (ROC) curve (AUC) for training and testing data was calculated as 0.866 and 0.568 , respectively. The important variable which contributed ratio in the modeling of $P$. papatasi was slope and NDVI factors. The results of jackknife test showed that the Bio8 (Mean Temperature of Wettest Quarter) factors with highest gain in the predicting power of the model if used in isolation (Fig. 7B). 

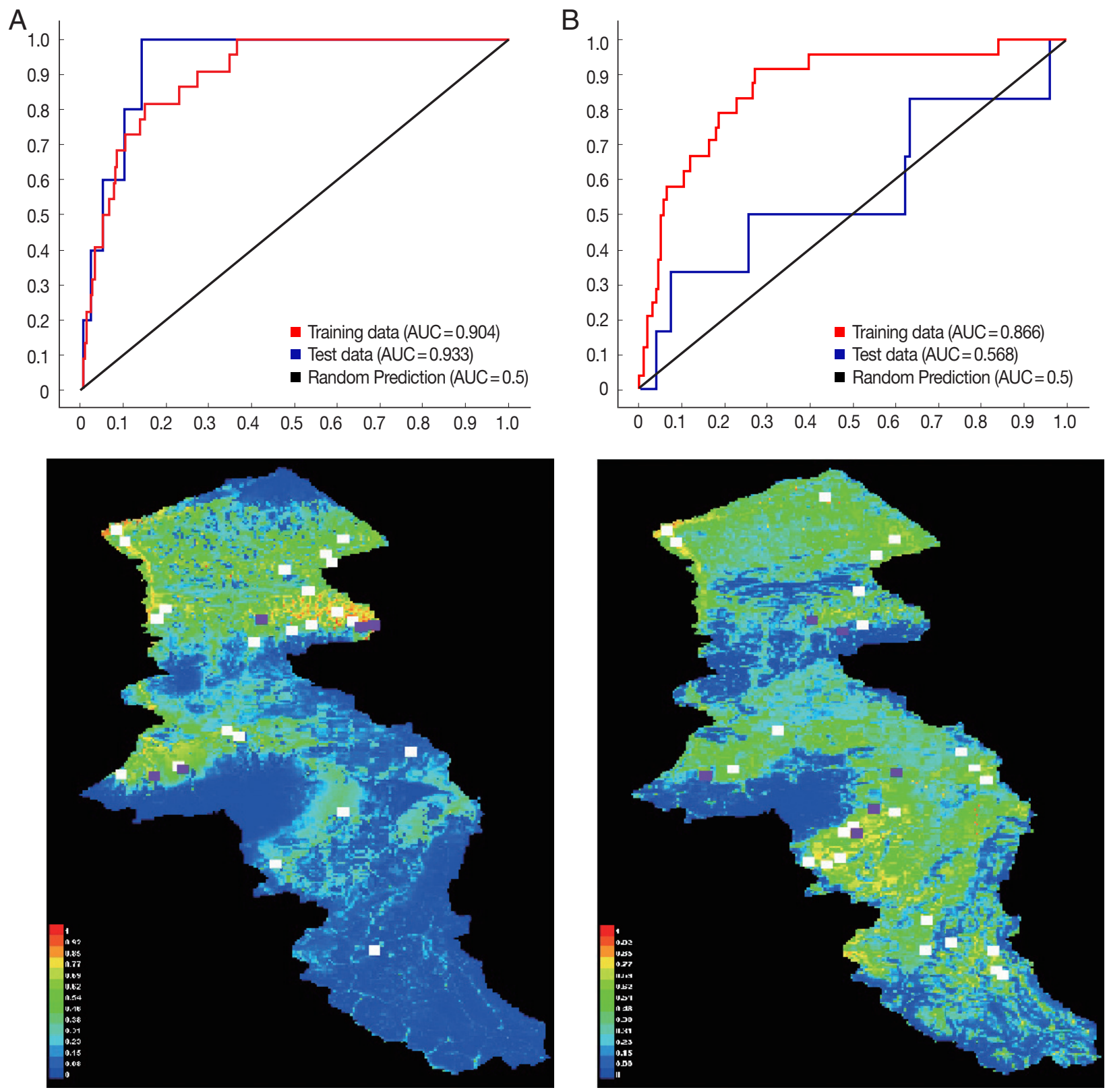

Fig. 6. Presentation of MaxEnt model (Upper) and receiver operating characteristic (Down) of two vectors of cutaneous leishmaniasis in northwest Iran. (A) Phlebotomus sergenti. (B) Phlebotomus papatasi.

\section{DISCUSSION}

Annually, 20-30 cases of CL were reported from Ardabil province and in $47 \%$ of the cases showed no history of travel outside the province. Out of human cases, $17 \%$ were under 10 years old and $74.5 \%$ of CL reported from 13 urban areas as well as $65 \%$ of lesions were dry and without secretion that these symptoms are probably related to ACL. The important clinical symptoms for ACL including dry ulcer that most occur in face in urban areas [17].

In The results of this study show that dominant species of Ardabil province is a P. papatasi. In a study which was completed by Absavaran et al. [19], Dehkordi et al. [22], Ghorbani et al. [23], and; Moradi-Asl et al. [24] confirmed the current results. P. papatasi and P. sergenti are the main vectors of ZCL and ACL in Iran respectively[3]. In this study $P$. papatasi was collected from all regions of Ardabil Province with a high frequency in north and central parts and P. sergenti from north part of Ar- 

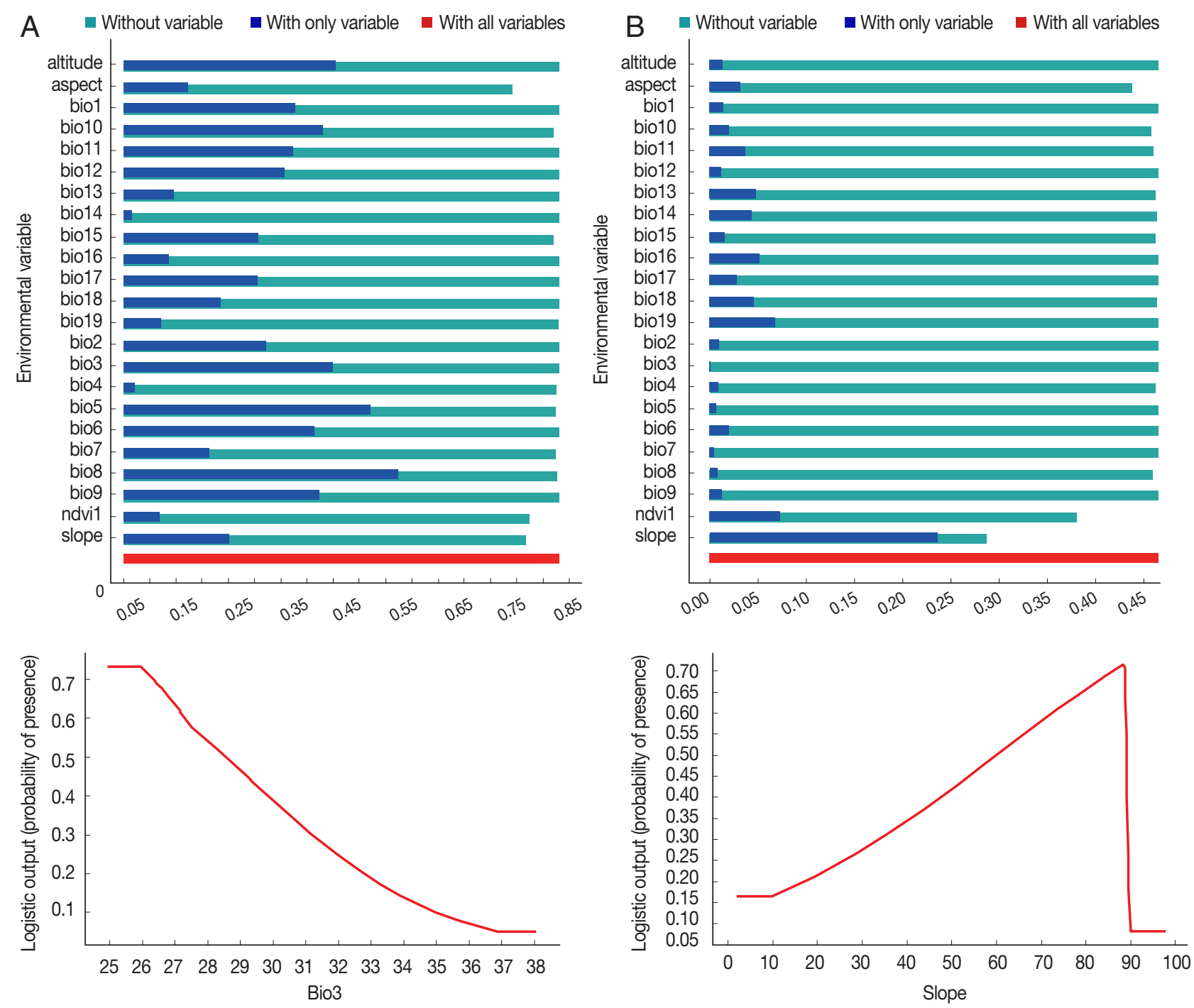

Fig. 7. Variable importance (upper) and the most important variable by jackknife test (down) for two vectors of cutaneous leishmaniasis in Ardabil Province. (A) Phlebotomus sergenti. (B) Phlebotomus papatasi.

dabil province, the same areas of CL reports in Bilasavar and Germi counties. This result showed that the vectors of CL is present in the Ardebil province with activity in June to July. Since $45 \%$ of $P$. papatasi and $24.5 \%$ of $P$. sergenti were collected from indoors, it seems that the sand flies have a feeding and resting in indoors places but invers, $P$. sergenti is more likely to outdoors places. In study of central Iran, 52\% of collected sand flies were P. papatasi that $33 \%$ of them were captured in indoor places and the seasonal activity was reported May to September [25]. The average temperature and altitude level in the central and northwest of Iran has a significant difference and have a greatest impacts on seasonal activity and distribution of sand flies [17-26]. The results of this study showed that the suitable habitat for $P$. sergenti in Ardabil Province were located in north part that this area have a lower than $1,500 \mathrm{~m}$ altitude. The results of MaxEnt model and jacknife test showed that isothermality and slope factors had the greatest effect on the distribution of $P$. sergenti in north part of Ardabil Province, respectively. With the slope increasing from 10 to 88 degrees, also frequency of $P$. papatasi has increased and after the $89 \mathrm{de}-$ grees the frequency of this spices has decreased. In one study, Lutzomyia whitmani the vector for CL in Southern Brazil was affected the east slope of the Andes [27] and the results of ecological niche model of $P$. alexandri and $P$. papatasi in the Middle East showed that elevation, minimum temperature in the coldest month, mean temperature in the wettest quarter, and 
precipitation in the driest month were the most important role in modeling of this spices [28]. For P. papatasi in Ardabil Province the suitable habitat was identified in north and central parts. The important factors that impacted on ecological niche model of $P$. papatasi were slope and NDVI. In central of Iran, lowland areas provides good ecological niches for $P$. papatasi and max-min temperature were impacted on ecological niche model [16]. According to the findings, cutaneous leishmaniasis might be endemic in Ardabil province with low incidence. Moreover, $P$. papatasi and $P$. sergenti as vectors of $\mathrm{CL}$ are present in northern and central parts of the province, and there are the potential of transmission of $\mathrm{CL}$ in the areas. It is recommended to more studies be done in the area in respect of $\mathrm{CL}$, reservoir and vectors.

\section{ACKNOWLEDGMENTS}

The authors are grateful to all colleagues at the University of Medical Sciences and staff at the health centers in the all counties in Ardabil Province. This study was funded Ardabil University of Medical Sciences (ARUMS), Project Number: 9601.

\section{CONFLICT OF INTEREST}

The authors declare no conflict of interest related to this study.

\section{REFERENCES}

1. Alvar J, Vélez ID, Bern C, Herrero M, Desjeux P, Cano J, Jannin J, den Boer M. Leishmaniasis worldwide and global estimates of its incidence. PLoS One 2012; 7: e35671.

2. World Health Organization. A Human Rights-Based Approach to Neglected Tropical Diseases [Internet]; Geneva, Switzerland; World Health Organization; [cited 2012 Sep 3]. Available from: https://www.who.int/gender-equity-rights/knowledge/ntd-information-sheet-eng.pdf?ua=1.

3. Yaghoobi-Ershadi M. Phlebotomine sand flies (Diptera: Psychodidae) in Iran and their role on Leishmania transmission. Iran J Arthropod Borne Dis 2012; 6: 1-17.

4. Yaghoobi-Ershadi MR. Control of phlebotomine sand flies in Iran: a review article. Iran J Arthropod Borne Dis 2016; 10: 429444.

5. Yaghoobi-Ershadi MR, Hanafi-Bojd AA, Javadian E, Jafari R, Zahraei-Ramazani AR, Mohebali M. A new focus of cutaneous leishmaniasis caused by Leishmania tropica. Saudi Med J 2002; 23: 291-294.

6. Javadian E, Nadim A, Tahvildare-Bidruni G, Assefi V. Epidemiol- ogy of cutaneous leishmaniasis in Iran: B. Khorassan Part V: Report on a focus of zoonotic cutaneous leishmaniasis in Esferayen. Bull Soc Pathol Exot Ses Fil 1976; 69: 140-143.

7. Nadim A, Tahvildari B. Epidemiology of cutaneous leishmaniasis in Iran: B. Khorassan. VI. Cutaneous leishmaniasis in Neishabur, Iran. Bull Soc Pathol Exot 1977; 70: 171-177.

8. Myler PJ, Fasel N. The biology of Leishmania-sand fly interactions. In Sacks D, Lawyer P, Kamhawi S eds, Leishmania: After the Genome.Norfolk, UK. Caister Academic Press. 2007; 205-238.

9. Rassi Y, Azizi K, Motazedian MH, Javadian E, Rafizadeh S, Fakhar M, Hatam GR. The seminested PCR based detection of Leishmania infantum infection in asymptomatic dogs in a new endemic focus of visceral leishmaniasis in Iran. Iran J Arthropod Borne Dis 2007; 1: 38-42.

10. Azizi K, Rassi Y, Javadian E, Motazedian MH, Rafizadeh S, Yaghoobi Ershadi MR, Mohebali M. Phlebotomus (Paraphlebotomus) alexandri: a probable vector of Leishmania infantum in Iran. Ann Trop Med Parasit 2006; 100: 63-68.

11. Karimi A,. Hanafi-Bojd, MR. Yaghoobi-Ershadi, Akhavan, Ghezelbash Z. Spatial and temporal distributions of phlebotomine sand flies (Diptera: Psychodidae), vectors of leishmaniasis, in Iran. Acta Trop 2014; 132: 131-139.

12. Moradi-Asl E, Hanafi-Bojd AA, Rassi Y, Vatandoost H, Mohebali M, Yaghoobi-Ershadi MR, Habibzadeh S, Hazrati S, Rafizadeh S. Situational analysis of visceral leishmaniasis in the most important endemic area of the disease in Iran. Iran J Arthropod Borne Dis 2017; 11: 482-492.

13. Mohebali M. Visceral leishmaniasis in Iran: review of the epidemiological and clinical features. Iran J Parasitol 2013; 8: 348358.

14. Oshaghi MA, Ravasan NM, Javadian EA, Mohebali M, Hajjaran H, Zare Z, Mohtarami F, Rassi Y. Vector incrimination of sand flies in the most important visceral leishmaniasis focus in Iran. Am J Trop Med Hyg 2009; 81: 572-577.

15. Rassi Y, Abai MR, Oshaghi MA, Javadian E, Sanei A, Rafidzadeh S, Arzamani K. First detection of Leishmania infantum in Phlebotomus kandelakii using molecular methods in north-eastern Islamic Republic of Iran. East Mediterr Health J 2012; 18: 387-392.

16. Minahan J. Encyclopedia of the Stateless Nations: Ethnic and National Groups Around the World. 2nd ed. Santa Barbara, USA. Greenwood. 2016, pp 225-230.

17. Rassi Y, Hanafi-Bojd AA. Phlebotomine Sand Flies, Vectors of Leishmaniases: Morphology, Biology, Ecology, and Field and Laboratory Methods with Pictorial Key of Iranian Sand Flies. Tehran, Iran. Noavaran-Elm Publication. 2006, pp 1-251 (in Persian).

18. Theodor O, Mesghali A. On the phlebotominae of Iran. J Med Entomol 1964; 1: 285-300.

19. Akhavan AA, Mirhendi H, Khamesipour A, Alimohammadian MH, Rassi Y, Bates P, Kamhawi S, Valenzuela JG, Arandian MH, Abdoli H, Jalali-zand N, Jafari R, Shareghi N, Ghanei M, Yaghoobi-Ershadi MR. Leishmania species: detection and identification by nested PCR assay from skin samples of rodent reservoirs. 
Exp Parasitol 2010; 126: 552-556.

20. Mohammadi AM, Khamesipour A, Khatami A, Javadi A, NassiriKashani M, Firooz A, Dowlati Y, Behnia M, Eskandari SE. Cutaneous leishmaniasis in suspected patients referred to the center for research and training in skin diseases and leprosy, Tehran, Iran from 2008 to 2011. Iran J parasitol 2013; 8: 430-436.

21. Absavaran A, Rassi Y, Parvizi P, Oshaghi MA, Abaie MR, Rafizadeh S, Mohebali M, Zarea Z, Javadian E. Identification of sand flies of the subgenus Larroussius based on molecular and morphological characters in North Western Iran. Iran J Arthropod Borne Dis 2009; 3: 22-35.

22. Dehkordi AS, Rassi YA, Oshaghi MA, Abai MR, Rafizadeh S, Yaghoobi-Ershadi MR, Mohebali M, Zarei Z, Mohtarami F, Jafarzadeh B, Ranjbarkhah A. Molecular detection of Leishmania infantum in naturally infected Phlebotomus perfiliewi transcaucasicus in Bilesavar district, northwestern Iran. Iran J Arthropod Borne Dis 2011; 5: 20-27.

23. Ghorbani E, Rassi Y, Abai M, Akhavan AA. Fauna and monthly activity of sand flies at endemic focus of visceral leishmaniasis in the west territory compared to the east of Meshkinshahr district, Ardebil Province. Iran J Public Health 2014; 12: 97-109.

24. Moradi-Asl E, Rassi Y, Adham D, Hanafi-Bojd AA, Saghafipour A, Rafizadeh S. Spatial distribution of sand flies (Diptera: Psychodidae; Larroussius group), the vectors of visceral leishmaniasis in Northwest of Iran. Asian Pac J Trop Biomed 2018; 8: 425-430.

25. Saghafipour A, Vatandoost H, Zahraei-Ramazani AR, YaghoobiErshadi MR, Rassi Y, Shirzadi MR, Akhavan AA. Spatial distribution of phlebotomine sand fly species (Diptera: Psychodidae) in Qom province, central Iran. J med entomol 2016; 54: 35-43.

26. Ready PD. Biology of phlebotomine sand flies as vectors of disease agents. Annu Rev Entomol 2013; 58: 227-250.

27. Peterson AT, Shaw J. Lutzomyia vectors for cutaneous leishmaniasis in Southern Brazil: ecological niche models, predicted geographic distributions, and climate change effects. Int J parasitol 2003; 33: 919-931.

28. Colacicco-Mayhugh MG, Masuoka PM, Grieco JP. Ecological niche model of Phlebotomus alexandri and P. papatasi (Diptera: Psychodidae) in the Middle East. Int J Health Geogr 2010; 9: 2-9. 